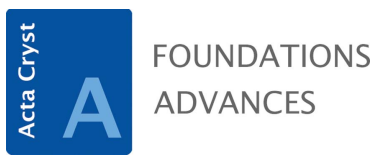

ISSN 2053-2733

\section{Crystallography: A Very Short Introduction. By A. M. Glazer. Oxford University Press, 2016. Pp. 168. Price GBP 7.99. ISBN 9780198717591.}

\author{
Claude Lecomte*
}

Université de Lorraine, CRM2, UMR 7036, Vandoeuvre-les-Nancy, F-54506, France, and CNRS, CRM2, UMR 7036, Vandoeuvre-les-Nancy, F-54506, France. *Correspondence e-mail: claude.lecomte@crm2.uhp-nancy.fr

This book is a new volume published as part of the Oxford 'Very Short Introduction' series, which aims to give easy access to various subjects. After a very general preface, which introduces the role of crystallography in science, this 168-page book is divided into six chapters.

Chapter 1 entitled 'A long history' is a very well documented chapter and may be the best chapter of the book; it clearly defines most concepts and all the steps in building the science of crystallography from Kepler's 1611 pamphlet to modern X-ray crystallography: symmetry, X-ray diffraction, mineral, organic and protein structures. I just regret that the author gives the example of the quartz prism to highlight sixfold (Fig. 3) symmetry: it is not false but it may cause the reader to think that quartz crystallizes in a hexagonal point group (unless he describes the high-temperature phase!). This historical chapter is also interesting because it briefly describes the life of most of the important pioneers of the field in relation to their research: for example, the author relates the strong opposition to W. L. Bragg's model of the NaCl structure (1915) and to the ionic bonding (p. 19) by reproducing an amazing letter published in Nature in 1927.

Chapter 2 is devoted to symmetry. It is a very short chapter, well written and without any mathematics; symmetry concepts, point groups and Bravais lattices are clearly introduced. Crystal structure and space groups are described using the convolution tool. However, it is surprising that the author never cites the Hermann-Mauguin space-group symbol which is used in Volume A of International Tables for Crystallography; he seems to prefer the term 'International Notation', but the Schoenflies symbol is also recognized as an international notation. Chapter 3 describes the crystal-structure types from close packing to protein structures. The paragraph entitled 'Close packing' in fact describes the most important inorganic structure types and some of their symmetry-related properties like piezoelectricity. I liked the discussion about the Bravais lattices of simple crystal structures. Polymorphism is introduced for the element carbon but is not discussed in the 'Organic structures' paragraph, which is a real pity: this paragraph is really too short and not at all up to date. It does not describe the key role of polymorphism in the pharmaceutical industry, in material science and in crystal-growth research; even the new, important concepts of crystal engineering and co-crystals are not discussed. As the book describes the physical properties of some minerals, a quick survey of the physical properties of the molecular materials (magnetism, optics, dielectrics etc.) would have been very welcome. This remark holds also for the paragraph 'Crystal growth', which is devoted to mineral materials and proteins. Biological molecules (proteins, virus, DNA) are described in more detail.

Chapters 4, 5 and 6 describe diffraction methods using X-rays (conventional tube, synchrotron and free-electron laser), neutrons and electrons. Chapter 4 introduces the diffraction concepts using a purely geometrical approach: first, the reciprocal lattice is defined and abruptly used to explain the Ewald sphere concept knowing the Bragg law (introduced much earlier, p. 15). Then the diffraction pattern is explained using the convolution theorem, but nothing is written about the interaction between matter and $\mathrm{X}$-rays, neutrons or electrons. I think that it is extremely important to show the reader that X-rays, neutrons or electrons are, respectively, scattered by the electron density, the nuclear and magnetic density or the electrostatic potential of the atoms [or generated by 
them for $V(r)$ ], because this is key for crystal-structure solutions and more advanced methods like electron-density modelling or magnetic structures. Chapter 4 ends with a description of incommensurate structures, quasicrystals and disorder based on the observation of their diffraction patterns. It is a rather long but interesting approach which shows the importance of careful experiments and interpretations. Disorder and quasicrystals are particularly well introduced. The example of the disorder of the aspirin molecule is well chosen, but it is the only one related to molecular crystals. Chapter 5 is entitled 'Seeing the atoms'; it is a 12-page chapter mostly describing the phase problem and the associated solutions (Patterson, direct and the recent charge-flipping methods), and its relation to the electron or nuclear densities. Generation of the structure of a naphthalene crystal by Fourier transform is well illustrated by Fig. 33. Refinement processes and the resulting quality of crystal structures are not mentioned, and I regret that this chapter does not go a step further by giving an example of modern electron-density modelling. A paragraph on the importance of crystallography databases would have been welcome because these big data drive modern research in crystallography. Chapter 6 describes and enumerates the sources of radiation. First, X-ray tubes, synchrotron radiation and free-electron lasers are described with their recent applications and possible future trends; this is followed by neutron diffraction to emphasize its importance for hydrogen-atom positions and magnetic structure determinations. The last paragraph is a very short introduction to electron diffraction.

In conclusion, this easy-to-read 'Short Introduction' is a description of crystallography and its applications. It is a good introduction for students and the public. Applications to minerals, ionic materials and proteins are well described but the strong contribution of crystallography to molecular materials and drug-design research is clearly missing, as well as big crystallographic data utilization. 